\title{
Enhanced antitumor and anti-angiogenic effects of metronomic Vinorelbine combined with Endostar on Lewis lung carcinoma
}

Rong-Sheng Qin ${ }^{1+}$, Zhen-Hua Zhang ${ }^{2+}$, Neng-Ping Zhu ${ }^{2+}$, Fei Chen ${ }^{1}$, Qian Guo ${ }^{1}$, Hao-Wen Hu' ${ }^{1}$ Shao-Zhi Fu ${ }^{2}$, Shan-Shan Liu', Yue Chen ${ }^{3}$, Juan Fan ${ }^{2 *}$ and Yun-Wei Han ${ }^{2 *}$

\begin{abstract}
Background: Conventional chemotherapy is commonly used to treat non-small cell lung cancer (NSCLC) however it increases therapeutic resistance. In contrast, metronomic chemotherapy (MET) is based on frequent drug administration at lower doses, resulting in inhibition of neovascularization and induction of tumor dormancy. This study aims to evaluate the inhibitory effects, adverse events, and potential mechanisms of MET Vinorelbine (NVB) combined with an angiogenesis inhibitor (Endostar).

Methods: Circulating endothelial progenitor cells (CEPs), apoptosis rate, expression of CD31, vascular endothelial growth factor (VEGF), hypoxia inducible factor-1 (HIF-1a) were determined using flow cytometry, western blot analysis, immunofluorescence staining and Enzyme-linked immunosorbent assay (ELISA) analysis. And some animals were also observed using micro fluorine-18-deoxyglucose PET/computed tomography $\left({ }^{18} \mathrm{~F}\right.$-FDG PET/CT) to identify changes by comparing SUVmax values. In addition, white blood cell (WBC) counts and H\&E-stained sections of liver, lungs, kidney, and heart were performed in order to monitor toxicity assessments.

Results: We found that treatment with MET NVB + Endo was most effective in inhibiting tumor growth, decreasing expression of CD31, VEGF, HIF-1a, and CEPs, and reducing side effects, inducing apoptosis, such as expression of BCl-2, Bax and caspase-3. Administration with a maximum tolerated dose of NVB combined with Endostar (MTD NVB + Endo) demonstrated similar anti-tumor effects, including changes in glucose metabolism with micro fluorine18-deoxyglucose PET/computed tomography $\left({ }^{18} \mathrm{~F}\right.$-FDG PET/CT) imaging, however angiogenesis was not inhibited. Compared with either agent alone, the combination of drugs resulted in better anti-tumor effects.

Conclusion: These results indicated that MET NVB combined with Endo significantly enhanced anti-tumor and antiangiogenic responses without overt toxicity in a xenograft model of human lung cancer.
\end{abstract}

Keywords: Anti-angiogenesis, Endostar, Metronomic chemotherapy, Vinorelbine

\footnotetext{
* Correspondence: fj-joan@163.com; 530018842@qq.com

${ }^{\dagger}$ Rong-Sheng Qin, Zhen-Hua Zhang and Neng-Ping Zhu contributed equally to this work.

'Suining first people's hospital, Sichuan Province, Suining 629000, China

${ }^{2}$ Department of Oncology, the Affiliated Hospital of Southwest Medical

University, Sichuan Province, Luzhou 646000, China

Full list of author information is available at the end of the article
}

(c) The Author(s). 2018 Open Access This article is distributed under the terms of the Creative Commons Attribution 4.0 International License (http://creativecommons.org/licenses/by/4.0/), which permits unrestricted use, distribution, and reproduction in any medium, provided you give appropriate credit to the original author(s) and the source, provide a link to the Creative Commons license, and indicate if changes were made. The Creative Commons Public Domain Dedication waiver (http://creativecommons.org/publicdomain/zero/1.0/) applies to the data made available in this article, unless otherwise stated. 


\section{Background}

Lung cancer is one of the most commonly diagnosed malignant tumors and non-small cell lung cancer (NSCLC) accounts for approximately $80-85 \%$ of all lung cancer diagnosis. Lung cancer exhibits a high rate of mortality and is often not diagnosed at an early stage. Although conventional chemotherapy has beneficial therapeutic effects, a major limitation of this type of chemotherapy is that the side-effects of the drugs reduce the quality of a patient's life. Because drug resistance is commonly seen in cases with NSCLC, more effective treatment strategies should be explored.

Metronomic chemotherapy (MET) is defined as a therapeutic approach by chronic administration of chemotherapeutic agents at a relatively low and minimally toxic dose without a prolonged drug-free break [1]. The mechanism involved exerts its anti-tumor effects by anti-angiogenic mechanisms, for example by inducing endothelial cell apoptosis, or by reducing viable circulating endothelial progenitor cells (CEPs) [2]. Previous studies have suggested that MET may be a multi-targeted anti-tumor strategy that restores anti-tumor immunity and induces tumor dormancy. Tumor growth and metastasis by antagonizing angiogenesis are also inhibited [3]. Previous studies have shown that metronomic oral Vinorelbine can be safely used in elderly patients with advanced NSCLC, allowing for long-term disease stabilization combined with optimal patient compliance $[4,5]$. Together, these studies, including numerous preclinical and clinical trials, provide accumulative evidence that MET maintains the therapeutic response, minimizes relapse after conventional chemotherapy, and overcomes resistance [1-3].

The inhibitor of angiogenesis, Endostar, is a modified recombinant human endostatin that is derived from rat vascular endothelial tumor cells [6] and inhibits tumor endothelial cell proliferation, angiogenesis, and tumor growth. Antimitotics, including taxanes and vinca alkaloids are lead drugs for metronomic treatment as they inhibit angiogenesis through multiple mechanisms [7]. Previous studies have shown that antitumor drugs can inhibit tumor cell growth effects on different cell cycles, and may induce tumor cell apoptosis. Vinorelbine binds to tubulin, thereby preventing formation of the mitotic spindle, which leads to cell death [8]. Vinorelbine is a semisynthetic vinca alkaloid and, as an oral formulation, is favored in the chronic administration protocol of MET [4, 5, 9]. Given this major advantage and necessity when using oral chemotherapy drugs in the clinic or in preclinical MET treatment approaches [4, 5], Vinorelbine was chosen as the prime candidate in this study. Several studies have reported that anti-angiogenic drugs combined with metronomic chemotherapy is used in for the treatment of advanced NSCLC and being investigated in various types of cancer, including cancer of the prostate and breast [10-12]. However, it is unknown whether Endostar combined with MET NVB enhances anti-tumor and anti-angiogenic effects in advanced stages of NSCLC. We investigated whether Endostar combined with MET NVB or Endostar combined with MTD NVB is superior regarding anti-tumor effects. We hypothesized that Endostar combined with MET NVB enhanced anti-tumor and anti-angiogenic responses without overt toxicity in a mous xenograft model. To test this hypothesis, we employed a xenograft model to evaluate the role of metronomic Vinorelbine and/or Endostar on the growth and angiogenesis of implanted lung tumors. In addition, we evaluated adverse events induced by the different treatment methods. The goal of this preclinical study was to provide a novel scientific approach to guide future clinical work.

\section{Methods}

\section{Cell culture and chemicals}

The murine Lewis lung carcinoma (LLC) cell line was purchased from the cell resource center of Shanghai institute of life sciences, Chinese academy of sciences (from ATCC) and was maintained in RPMI-1640 medium, supplemented with $10 \%$ fetal calf serum, and the catalogue number of the cell lines used in this study was ATCC ${ }^{\oplus}$ Number: CRL-1642 ${ }^{\text {тм }}$. The recombinant human endostatin, Endostar, was provided by Shandong Simcere Medgenn Bio-pharmaceutical Co. Ltd. (Yantai, Shandong, China) and stored at $4{ }^{\circ} \mathrm{C}$ until required. The doses and the schedules of administration of reference drugs were based on previous studies [13, 14].

Injectable Vinorelbine solution was supplied by the Southwest Medical University (Luzhou, China). The dose of Vinorelbine was chosen based on what was previously described $[15,16]$. According to the dose conversion table for animal and human body weights, in which the Du Bois formula is used to calculate the body surface area (BSA) of the patient $\left(\mathrm{m}^{2}\right): 0.007184 \times(\text { patient height in } \mathrm{cm})^{0.725} \times($ patient weight in $\mathrm{kg})^{0.425}$, the MTD of Vinorelbine for mice was $10 \mathrm{mg} / \mathrm{kg}$, and the MET dose was the maximum daily dose of 1/10-1/3. Therefore, doses of $1.5,2,2.5,3$, and $3.5 \mathrm{mg} / \mathrm{kg}$ were chosen for the preliminary experiments. An optimal $3 \mathrm{mg} / \mathrm{kg}$ of Vinorelbine was chosen, based on the maximum antitumor effect and anti-angiogenesis effect (data not shown).

\section{Animals}

Female C57BL/6 J mice (3-4 weeks of age) were acclimatized for at least a week under standard conditions of 24 $\pm 2{ }^{\circ} \mathrm{C}$ and $50 \pm 10 \%$ relative humidity before they were enrolled in the study. All animals were sacrificed by orbital puncture at second days after treatment. The animal protocol used in this study was reviewed and approved by 
the Institutional Animal Care and Use Committee of the Southwest Medical University (Luzhou, China).

\section{Mouse xenograft model and treatments}

To establish a mouse xenograft model, a total of $1 \times 10^{6}$ LLC cells were resuspended in $0.1 \mathrm{ml}$ phosphate buffered saline (PBS; $\mathrm{pH}, 7.0$ ) and injected subcutaneously into the back of each animal near the right axilla. When the tumors reached a size of approximately $200 \mathrm{~mm}^{3}$, ninety tumor-bearing mice were randomized into six groups ( $n$ $=15$ mice per group) and treated for 14 consecutive days as follows: i) NS group (negative control), ii) Endostar group (10 mg/kg/day), iii) MET NVB group, metronomic Vinorelbine group, ( $3 \mathrm{mg} / \mathrm{kg}$ body weight (bw) of Vinorelbine every other day), i.v.) MET NVB + Endo group, v) maximum tolerated dose (MTD) NVB group, $(10 \mathrm{mg} / \mathrm{kg}$ body weight (bw) of Vinorelbine i.p. on days 1 and 8); and vi) MTD NVB + Endo group. All compounds were administrated intraperitoneally (i.p) and mimicked the oral metronomic administration. During the treatment period, tumors were measured every other day using calipers. The tumor volumes were calculated using the following formula: tumor volume $\left(\mathrm{cm}^{3}\right)=$ length $\times$ width ${ }^{2} \times 0.5$ and a tumor growth curve was plotted based on tumor size. The tumor growth inhibition rate on day 15 after treatment was determined using the following formula:

$$
\begin{aligned}
& \text { Inhibition rate }(\%)=(1-\mathrm{A} / \mathrm{B}) \times 100 \% \\
& \mathrm{~A}=\text { Volume }_{\text {Day1 experiment group }}-\text { Volume }_{\text {Day15 experiment (group) }} \\
& \mathrm{B}=\text { Volume }_{\text {Day1 control group }}-\text { Volume }_{\text {Day15 control group. }}
\end{aligned}
$$

\section{Flow cytometry}

A single cell suspension of $1 \times 10^{6}$ cells $/ \mathrm{ml}$ was prepared from isolated tumor tissue, and incubated for $15 \mathrm{~min}$ in the dark with $5 \mu \mathrm{l}$ Annexin V-FITC and $5 \mu \mathrm{l}$ PI. A total of $100 \mu \mathrm{l}$ of peripheral blood from each mouse was stained with CD133-FITC (1:20), CD34-APC (1:20), and Flk-1-PE (1:20), and incubated for $30 \mathrm{~min}$ in the dark. Next, red blood cells were lysed for $15 \mathrm{~min}$ and peripheral blood nuclear cells were collected. CD $133^{+} \mathrm{CD} 34^{+}$Flk- $1^{+}$cells represented the frequency of CEPs [17-20]. The frequency of CEPs and the apoptosis rate were determined by flow cytometry analysis (BD FACS Calibur, San Jose, CA, USA).

\section{Immunohistochemistry}

Tumors were fixed in 10\% neutral-buffered formalin solution, embedded in paraffin, and 4 um thick sections were cut for immunohistochemical analysis. Sections were stained with antibodies directed against CD31, VEGF, and HIF-1 $\alpha$ (1:100, Bioworld Technology, Louis Park, MN, USA), and were performed according to the manufacturer's instructions (Bioworld Technology, Louis Park,
MN, USA). Images were taken using an optical microscope (Olympus, Tokyo, Japan). Staining intensity was scored by two independent experienced pathologists. Each sample was graded according to intensity and extent of staining. The intensity of staining was scored as 0 (no staining), 1 (weak staining), and 2 (strong staining). The extent of staining was based on the percentage of positive tumor cells: 0 (no staining), 1 (1-25\%), 2 (26-50\%), 3 (51-75\%), and 4 (76-100\%). These two scores were added together for a final score. A case was considered negative if the final score was 0 or $1(-)$ or 2 or $3( \pm)$, and positive if the score was 4 or $5(+)$ or 6 or $7(++)$. In most cases, the two examiners provided consistent results. Any inconsistencies were resolved by discussion to achieve a consensus score.

\section{Western blot analysis}

Tumor samples were homogenized, and centrifuged at $12,000 \mathrm{rpm}$ for $15 \mathrm{~min}$ at $4{ }^{\circ} \mathrm{C}$. The protein concentration in supernatant was determined using a BCA colorimetric assay (Thermo Scientific Rockford, IL, USA). Approximately $40 \mu \mathrm{g}$ of the supernatant was resolved by SDS-PAGE analysis and transferred to nitrocellulose membranes. Membranes were blocked for $1 \mathrm{~h}$ with $5 \%$ nonfat milk in $1 \times$ PBS and incubated overnight at $4{ }^{\circ} \mathrm{C}$ with primary antibodies directed against VEGF Receptor 2, HIF-1 $\alpha$, Bcl-2, Bax, and caspase-3 (1:1000 dilution, Cell signaling Technology, Boston, MA, USA). Next, membranes were washed three times $10 \mathrm{~min}$ with $1 \times \mathrm{PBS}$ and incubated with a peroxidase-conjugated secondary antibody (1:3000 dilution, Cell signaling Technology, Boston, MA, USA) for $1 \mathrm{~h}$ under shaking at room temperature. After incubation, membranes were washed three times $10 \mathrm{~min}$ with $1 \times \mathrm{PBS}$ and proteins were visualized using chemiluminescence. GAPDH was used as an internal reference for protein loading. Signals were quantified using ImageQuant 5.0 software (Molecular Dynamics, Sunnyvale, CA, USA).

\section{Enzyme-linked immunosorbent assay analysis}

Roughly $1 \mathrm{ml}$ of peripheral blood from was collected by orbital puncture in Eppendorf tubes and allowed to naturally coagulate for 10 $20 \mathrm{~min}$ at room temperature. After centrifugation $(1800 \mathrm{~g})$ for $10 \mathrm{~min}$ at $4{ }^{\circ} \mathrm{C}$, the protein in the serum was precipitated and immediately frozen at $80{ }^{\circ} \mathrm{C}$ until further analysis. HIF- $1 \alpha$ and VEGF levels were determined by an ELISA kit according to the manufacturer's guidelines (Beijing Cheng Lin biological technology co, LTD, Beijing, China). A total of $10 \mu \mathrm{l}$ serum sample and $40 \mu \mathrm{l}$ of the standard solutions were added to the wells, incubated at $37{ }^{\circ} \mathrm{C}$ for $30 \mathrm{~min}$, and washed 5 times with diluted detergent solution. Subsequently, the wash solution was removed, and $50 \mu \mathrm{l}$ of Enzyme labeling reagent was added to each well, followed by incubation at $37{ }^{\circ} \mathrm{C}$ for $30 \mathrm{~min}$. Next, wells were washed 5 times and $50 \mu \mathrm{l}$ of stop 
solution was added to each well. The absorbance was read at a wavelength of $450 \mathrm{~nm}$ and HIF-1 $\alpha$ and VEGF concentrations were calculated using a standard curve.

\section{Micro ${ }^{18}$ F-FDG PET/CT imaging}

Positron emission tomography (PET) using 18F-FDG to monitor antitumor effects was used to identify changes in the glucose metabolism. To study the reactivity of tumor tissue in the experimental groups, we performed micro $\mathrm{PET} / \mathrm{CT}$ scans and image analysis the day after termination of treatment, using an Inveon micro PET/CT animal scanner (Siemens, Munich, Germany). Mice were fasted for $12 \mathrm{~h}$, and anesthetized with $1 \%$ pentobarbital $(5 \mathrm{ml} / \mathrm{kg})$, injected intravenously with 100-200 mCi FDG via the tail vein, and scanned. After roughly $40 \mathrm{~min}$, PET/CT images were acquired and collected for data analysis that was performed by comparing the maximum of standardized uptake value (SUVmax values).

\section{Evaluation of side effects and histopathological analysis}

Possible side effects were indicated by observation of body weight, diarrhea, and behavior. Peripheral blood was collected by orbital puncture using in heparin-coated tubes to prevent coagulation, and analyzed by the blood cell automatically detect analyzer (Mindray, Shenzhen,
China). After mice were sacrificed, liver, lungs, kidney, and heart were harvested for hematoxylin and eosin (H\&E) staining. H\&E-stained sections were visualized by two pathologists in a blinded manner.

\section{Statistical analysis}

Data are expressed as the mean \pm standard deviation. The statistical significance of the differences between treatment groups was determined by one-way analysis of variance (ANOVA) and the average number of pairwise comparisons was determined by Tamhane's T2 test. $P<0.05$ was considered statistically significant. Statistical analyses were performed using SPSS software version 19.0 (SPSS, Inc., Chicago, IL, USA).

\section{Results}

MET NVB combined with Endostar inhibits the growth of xenograft tumors in vivo

In Fig. 1a, tumor growth curves are presented. The data showed that in the control group the tumors grew rapidly, however, the tumor growth was significantly decreased in all treatment groups $(P<0.01)$. Compared with other groups, the tumor volumes in the MET NVB + Endo group were significantly smaller $(P<0.01)$, except for that of the MTD NVB + Endo group, which indicated that the
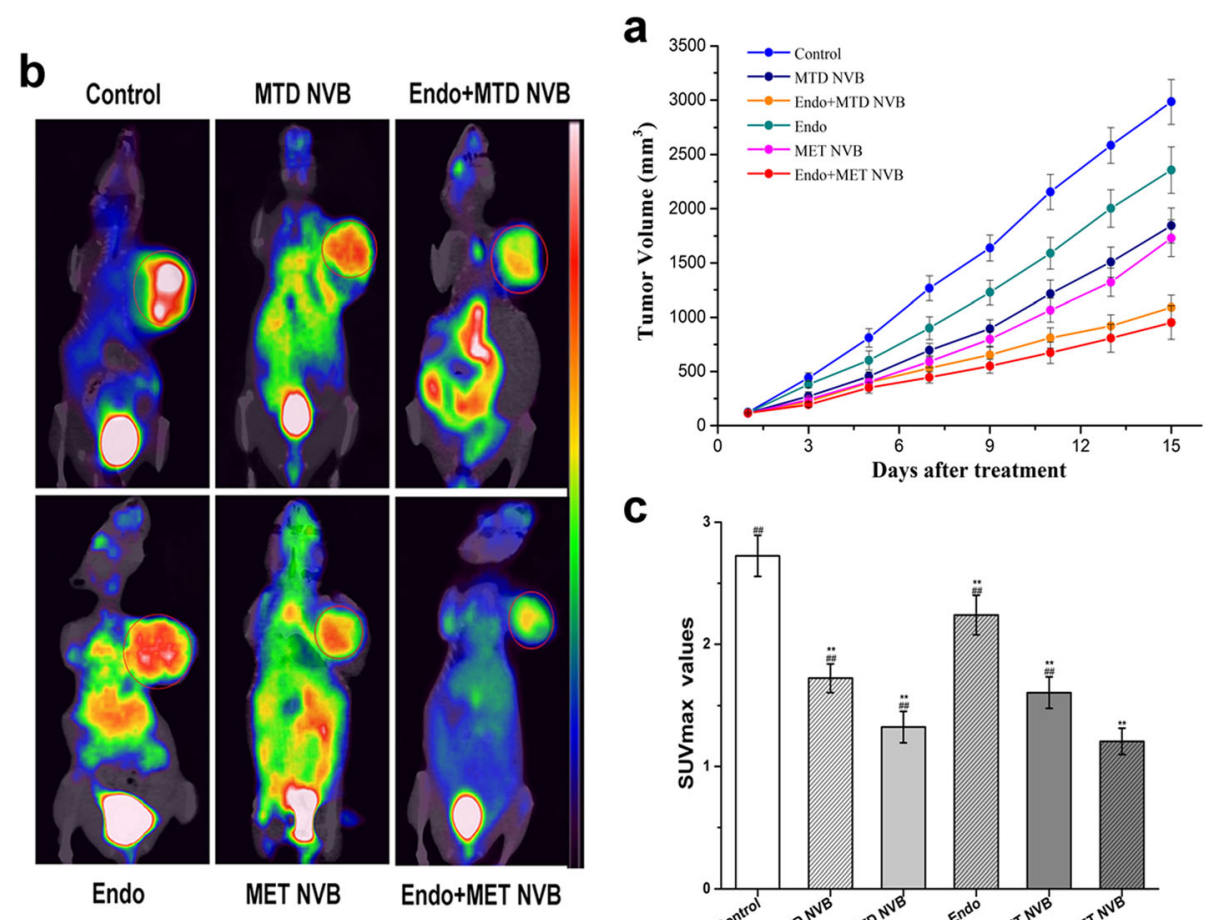

C

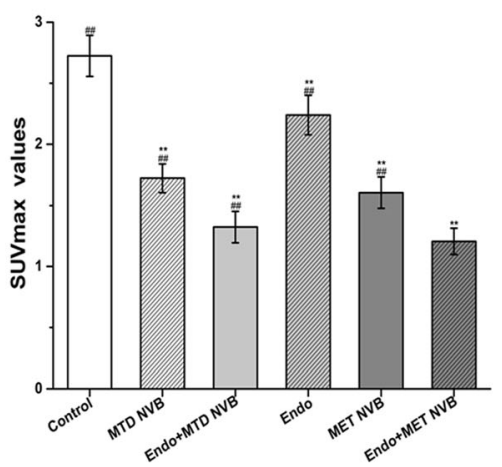

Fig. 1 MET NVB treatment combined with Endostar (Endo) inhibits the growth of xenograft tumors in mice. a Tumor growth curves in six groups ( $n=12$ for Control, MET NVB, Endo, MET NVB + Endo, MTD NVB, and MTD NVB + Endo groups). Representative ${ }^{18}$ F-FDG PET images (b) and SUVmax values (c) of mice after one full day of treatment. Data are expressed as the mean \pm SD. ${ }^{*} P<0.05,{ }^{* *} P<0.01$ versus the control group; ${ }^{\#} P<0.05$, ${ }^{\# \#} P<0.01$ versus the MET NVB + Endo group 
tumor size was not significantly different between the two groups $(P>0.05)$. Taken together, these data demonstrated that treatment with any drug inhibited the growth of xenograft tumors, and that the MET NVB + Endo group and MTD NVB + Endo group showed the greatest efficacy in tumor growth inhibition compared with other treatment groups $(P<0.05$ in all cases).

\section{Micro ${ }^{18}$ F-FDG PET imaging}

Representative ${ }^{18}$ F-FDG PET images and SUVmax values of mice in different groups are presented in Fig. 1b, c. The SUVmax value in the MET NVB + Endo treatment group was the lowest when compared to the SUVmax values of the other groups $(P<0.05)$. However, this difference was not significant when compared with the MTD NVB + Endo treatment group $(P>0.05)$. Additionally, both Vinorelbine alone groups and the Endo treatment group showed a decrease in SUVmax values compared to the control group $(P<0.05)$, however the difference between these three groups was not statistically significant. Taken together, these results indicated that the MET NVB + Endo treatment group had a similar effect as the MTD NVB + Endo group, and had an increased tumor growth inhibition effect compared with the other treatment groups tested.

\section{MET NVB combined with Endostar decreases the}

\section{frequency of peripheral blood CEPs}

We initially characterized the frequency of peripheral blood CEPs in the different treatment groups (Fig. 2a, b). Because the level of CEPs was low, no significant difference was observed in the total number of blood nuclear cells between groups. However, flow cytometry results showed that a significant different in the proportion of CEPs was found when comparing the MET NVB + Endo group with other groups $(P<0.05)$. In particular, the frequency of CEPs in the MET NVB + Endo group $(0.023 \pm 0.012 \%)$ was significantly lower compared to that of all other groups $(P<0.05)$. The frequency of CEPs in the MET NVB group and the Endo group were $0.035 \pm 0.01 \%$ and $0.04 \pm 0.016 \%$. Secondly, a relatively high proportion of peripheral blood CEPs was discovered in the ctrl group, the MTD NVB group, and the MTD NVB + Endo group. Interestingly, treatment of mice with MTD NVB or MTD NVB + Endo increased the frequency of total CEPs $(0.058 \pm 0.014 \%$ or $0.068 \pm$ $0.019 \%$, respectively, $P<0.01)$. Collectively, these data indicated that MET NVB or Endostar significantly decreased the frequency of peripheral blood CEPs, and that combined treatment of both further reduced this frequency in mice.

\section{MET NVB combined with Endostar reduces tumor- associated microvessel density}

As shown in Fig. 3a, immunohistochemical images showing CD31 expression changes in tumor tissue in different treatment groups showed that a high level of MVD was found in the MTD NVB + Endo group, MTD NVB group, and ctrl group, whereas a lower MVD value was observed in the MET NVB group, Endo group, and MET NVB + Endo group. As shown in Fig. 3b, quantitative analysis of CD31 expression in tumor tissue indicated that MVDs from the MET NVB group $(4.17 \pm 0.75)$ were different

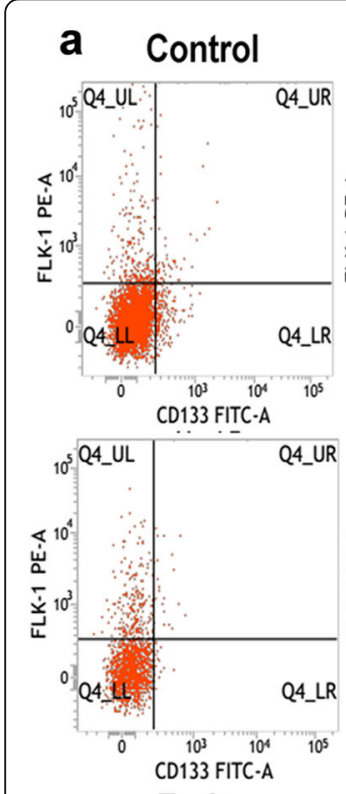

Endo
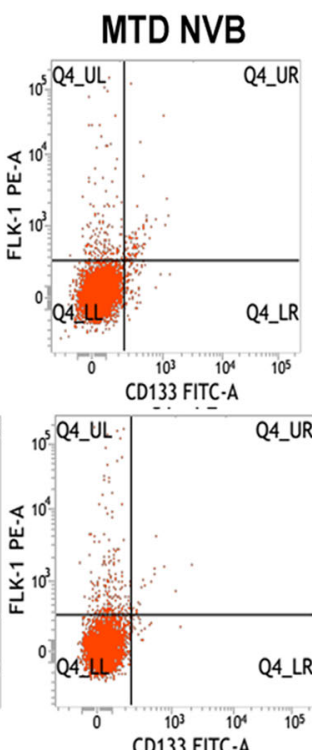

MET NVB

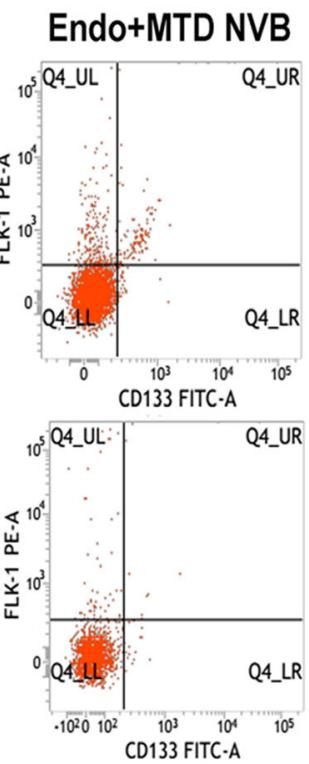

Endo+MET NVB

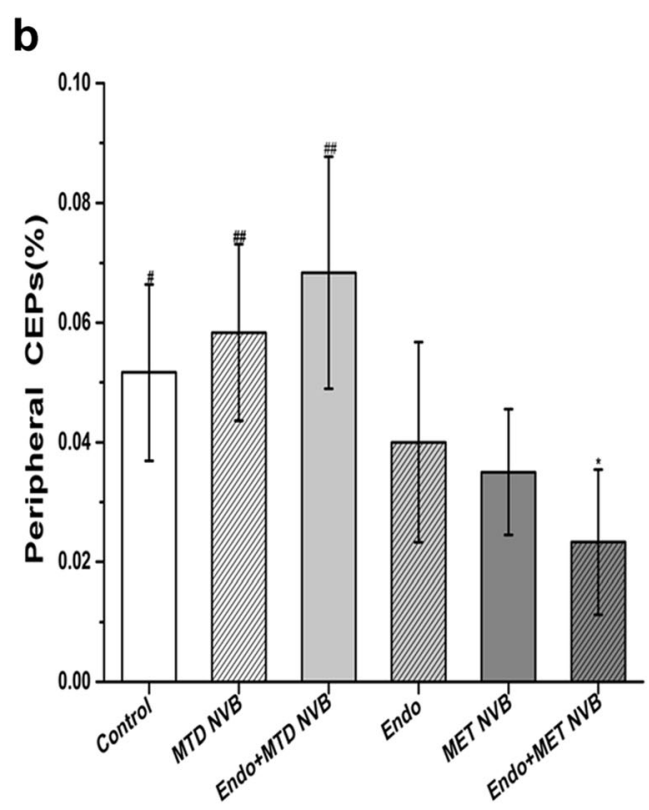

Fig. 2 MET NVB treatment combined with Endostar (Endo) decreases the frequency of peripheral blood CEPS. a The frequency of peripheral blood CEPs in different groups was determined by flow cytometry analysis. $\mathbf{b}$ Histogram showing the quantitative data of the mean frequency of CEPs per treatment group. Data are expressed as the mean \pm SD. ${ }^{*} P<0.05,{ }^{* *} P<0.01$ versus the control group; ${ }^{\#} P<0.05,{ }^{\# \#} P<0.01$ versus the MET NVB + Endo group 

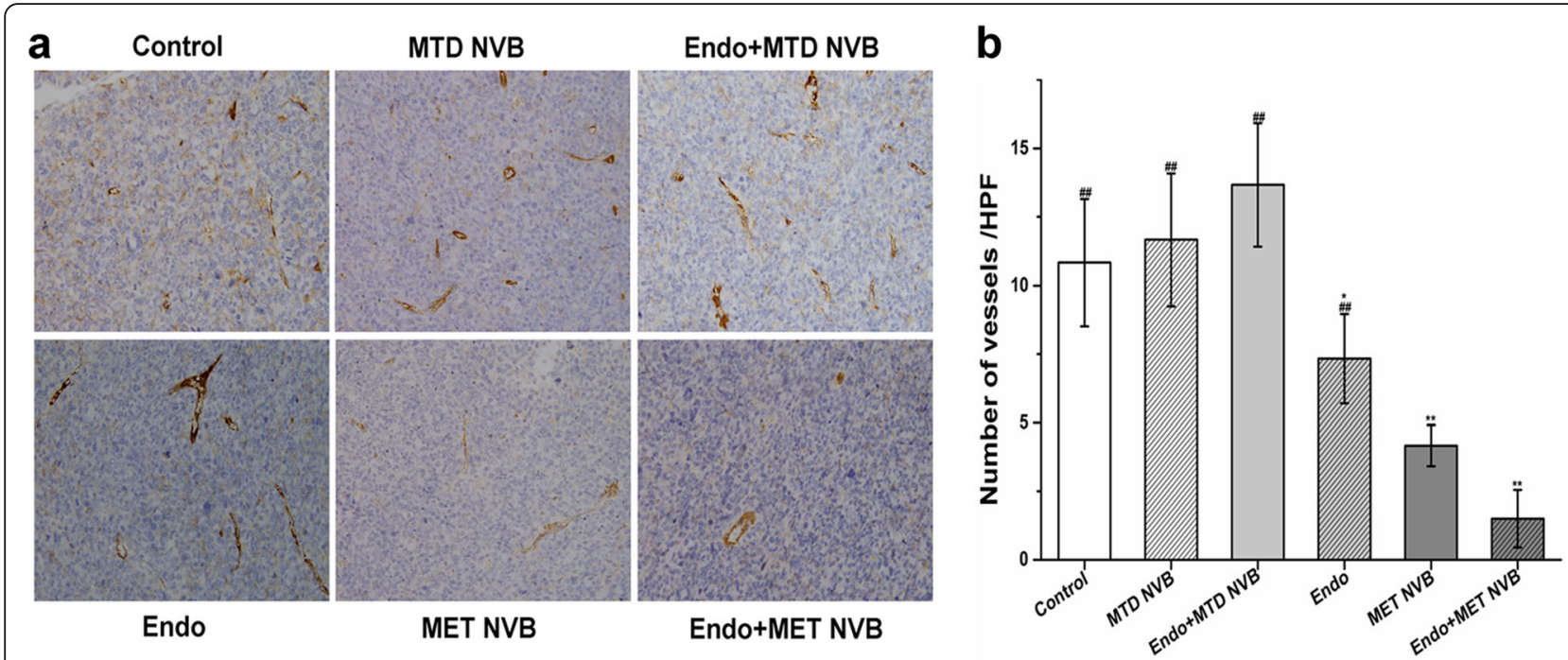

Fig. 3 a Immunohistochemical images showing CD31 expression changes in tumor tissue in different treatment groups. b Quantitative analysis of CD31 expression in tumor tissue of xenograft mice in different groups. Data are expressed as the mean $\pm \mathrm{SD} .{ }^{*} P<0.05,{ }^{* *} P<0.01$ versus the control group; ${ }^{\#} P<0.05,{ }^{\# \#} P<0.01$ versus the MET NVB + Endo group. Original magnification, $\times 400$

from that of the Endo-treated group of mice $(7.33 \pm 1.63$, $P<0.05)$, and both were greater than that in the MET $\mathrm{NVB}+$ Endo group $(1.50 \pm 1.05, \mathrm{P}<0.05)$. A relatively high level of CD31 expression in tumor tissue was discovered in the ctrl group $(10.83 \pm 2.32)$, the MTD NVB group $(11.67 \pm 2.42)$, and the MTD NVB + Endo group $(13.67 \pm$ 2.25). Furthermore, differences between the MET NVB + Endo group of mice were particularly notable when compared with all other groups $(P<0.01$ vs. other groups). Therefore, these data suggested that MET NVB or Endostar, significantly inhibited the formation of microvascular vessels in xenograft tumors.

\section{MET NVB combined with Endostar decreases expression of VEGF and HIF-1a}

To further verify our findings, ELISA analysis was performed. As shown in Fig. 4b, in the MET NVB + Endo group the serum level of VEGF was $76.52 \pm 9.25 \mathrm{pg} / \mathrm{ml}$ (P $<0.01$ vs. other groups), whereas in the ctrl group showed serum VEGF levels of $227.3 \pm 8.55 \mathrm{pg} / \mathrm{ml}$. Moreover, in the MTD NVB + Endo group, the VEGF serum level was $240.54 \pm 11.29 \mathrm{pg} / \mathrm{ml}$. Consistent with the results obtained by the ELISA assay, the expression of VEGF and HIF- $1 \alpha$ as determined by immunohistochemical analyses were shown in Fig. 4a and d. Compared with the Ctrl group, the expression of VEGF were reduced in the MET NVB group and Endo group, further significantly reduced in the MET NVB + Endo group. However, higher expression levels of VEGF and HIF- $1 \alpha$ were observed in the MTD NVB and MTD NVB + Endo group. As shown in Fig. 4e and Table 1, VEGF protein levels as determined using western blot analysis were decreased in the MET NVB + Endo group, but increased in MTD NVB + Endo group.
We observed a similar trend of changes for the expression of HIF- $1 \alpha$. In conclusion, we found a reduced expression of VEGF and HIF-1 $\alpha$ in the MET NVB + Endo group and a higher expression of VEGF and HIF- $1 \alpha$ in the MTD $\mathrm{NVB}+$ Endo group.

\section{MET NVB combined with Endostar increases the apoptosis rate of tumor tissue}

The flow cytometry analyses presented in Fig. 5a, b show that apoptosis rates of the chemotherapy alone treatment groups was very low compared with that of combined treatment groups $(P<0.05)$. Moreover, the apoptosis rate of tumor tissue was significantly increased $(P<0.05)$ after treatment with MET NVB + Endo and MTD NVB + Endo $(P>0.05)$, indicating that treatment with MET NVB + Endo and MTD NVB + Endo had similar effects. Surprisingly, a higher apoptosis rate of tumor tissue was observed in the ctrl group. To assess the effect of the apoptosis rate, we further determined the expression of Bcl-2, Bax, and caspase- 3 by Western blot analysis (Fig. $5 \mathrm{c}$ and Table 2), which indicated that the amount of Bax and caspase- 3 protein was increased, but the $\mathrm{Bcl}-2$ protein level was decreased in the MET NVB + Endo group and MTD $\mathrm{NVB}+$ Endo group. Taken together, quantitative analyses indicated that treatment with MET NVB + Endo and MTD NVB + Endo significantly induced apoptosis and caused a synergistic effect.

\section{Toxicity assessments}

To investigate the side effects induced by the different therapeutic regimens, we collected liver, lungs, kidney, and heart tissue and blood to analyze changes in white blood cell (WBC) counts. As shown in Fig. 6, in MTD 


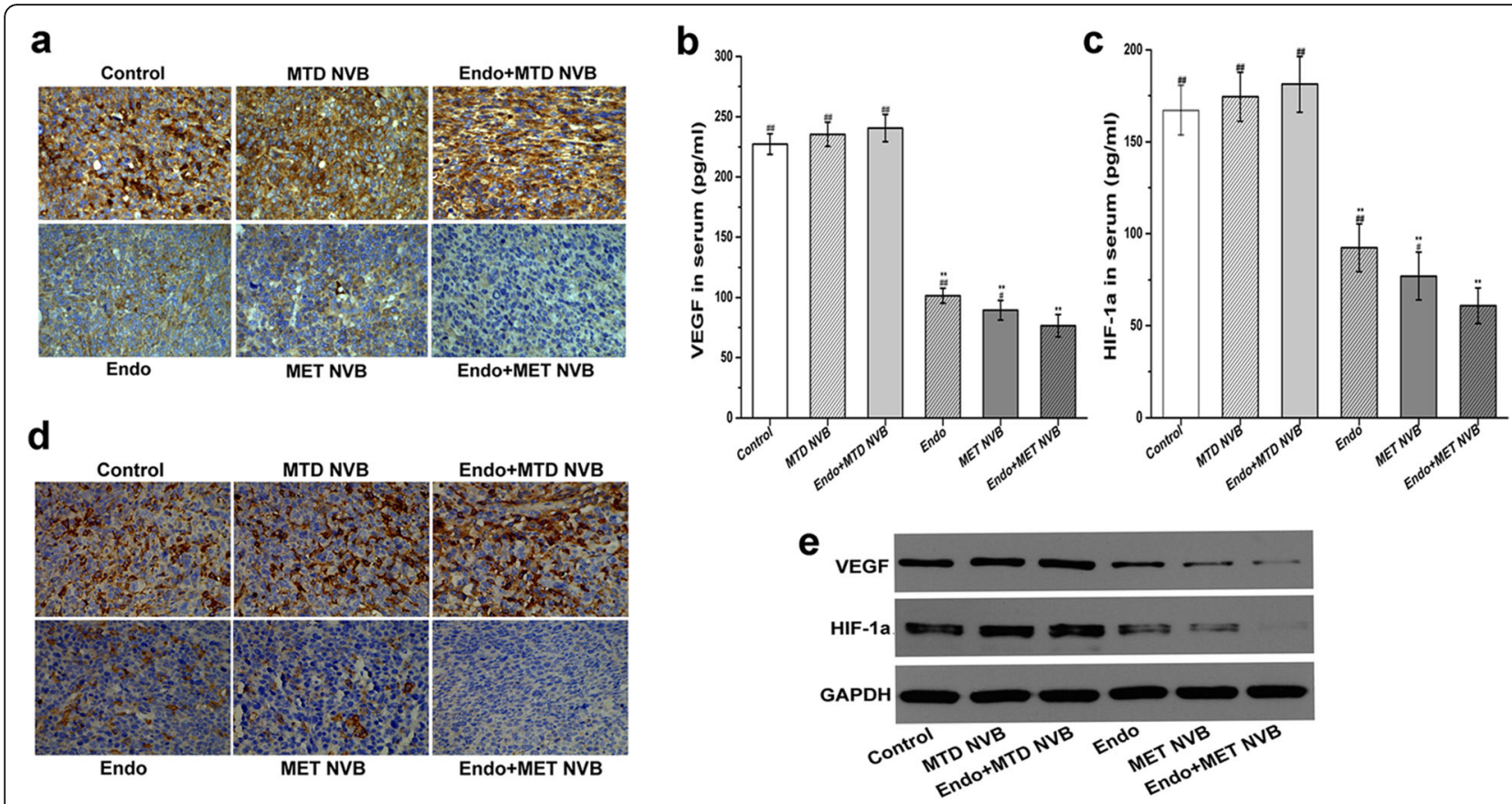

Fig. 4 a Immunohistochemical analysis showing expression of VEGF in different treatment groups. b, c Serum levels of VEGF and HIF-1a determined by ELISA assay in different treatment groups. $\mathbf{d}$ Representative images of immunohistochemical analysis showing expression of HIF-1a in different treatment groups. e Expression of VEGF and HIF-1a was determined by Western blot analysis. GAPDH served as the loading control. Data are presented as the mean $\pm \mathrm{SD} .{ }^{*} P<0.05,{ }^{* *} P<0.01$ versus the control group; ${ }^{\#} P<0.05,{ }^{\# \#} P<0.01$ versus the MET NVB+ Endo group

NVB or MTD NVB + Endo groups WBC counts were $(4.3 \pm 1.48) \times 10^{3}$ number/ $\mu \mathrm{l}$, or $(4.23 \pm 1.86) \times 10^{3}$ number/ $\mu \mathrm{l}$ (respectively). However, in ctrl group, the WBC count was $(9.55 \pm 2.2) \times 10^{3}$ number/ $\mu \mathrm{l}$, and in the MET $\mathrm{NVB}$ or MET NVB + Endo group, the WBC counts were $(8.41 \pm 2.32) \times 10^{3}$ number/ $\mu \mathrm{l}, \quad(8.26 \pm 1.23) \times 10^{3}$ number/ $\mu$ l (respectively, $P>0.05$ ). These results showed that WBC counts were reduced in the MTD NVB group, while in the MET NVB group WBC counts were within the normal range. H\&E-stained sections of the liver, lungs, kidney, and heart of each group were visualized using a light microscope. The H\&E-stained sections of the MTD NVB group showed chronic inflammation and interstitial thickening of lung tissue, and hepatic cell edema, degeneration, necrosis and hepatic structural disorders in liver tissue. This was not found it sections

Table 1 Expression of VEGF and HIF-1a in each group

\begin{tabular}{lll}
\hline Groups & VEGF/GAPDH & HIF-1a/GAPDH \\
\hline Control & $0.515 \pm 0.002^{\# \#}$ & $0.555 \pm 0.002^{\# \#}$ \\
MTD NVB & $0.547 \pm 0.003$ & $0.754 \pm 0.003$ \\
Endo+MTD NVB & $0.639 \pm 0.004^{\# \#}$ & $0.868 \pm 0.004^{\# \#}$ \\
Endo & $0.346 \pm 0.001$ & $0.382 \pm 0.002$ \\
MET NVB & $0.181 \pm 0.001$ & $0.201 \pm 0.001$ \\
Endo+MET NVB & $0.078 \pm 0.001^{* *}$ & $0.041 \pm 0.001^{* *}$ \\
\hline
\end{tabular}

Data are presented as the mean \pm SD. ${ }^{*} P<0.05,{ }^{* *} P<0.01$ versus the control group; ${ }^{\#} P<0.05,{ }^{\# \#} P<0.01$ versus the MET NVB+ Endo group derived from the MET NVB group. H\&E-stained sections showing macroscopic metastasis of lung and liver tissue were only found in the ctrl group. No organizational changes were found in kidney and heart tissue, and no differences were found between groups.

\section{Discussion}

Advanced stage tumors are not effectively eradicated by conventional chemotherapy because of suboptimal drug targeting, the onset of therapeutic resistance, side effects, and neoangiogenesis. Therefore, novel strategies are required for chemosensitization of cancer cells. MET refers to the close, regular administration of conventional chemotherapy drugs at relatively low, minimally toxic doses with no prolonged break periods [21]. MET is thought to primarily cause antitumor effects by anti-angiogenic activities, both locally by targeting endothelial cells of the tumor neovasculature, and systemically by acting on bone marrow-derived cells, including CEPs [21, 22]. Previous studies have shown that after giving a tumor-burdened mouse a vascular breaker, CEPs can rapidly be mobilized and participate in the regeneration of tumor-associated blood vessels [23, 24]. In a phase III clinical trial of endostatin (ES) in China, the combination of ES and chemotherapy significantly improved the overall and progression-free survival of patients with advanced NSCLC [25]. The combined drug delivery approach led us to hypothesize that metronomic administration of joint anti-angiogenesis drugs 


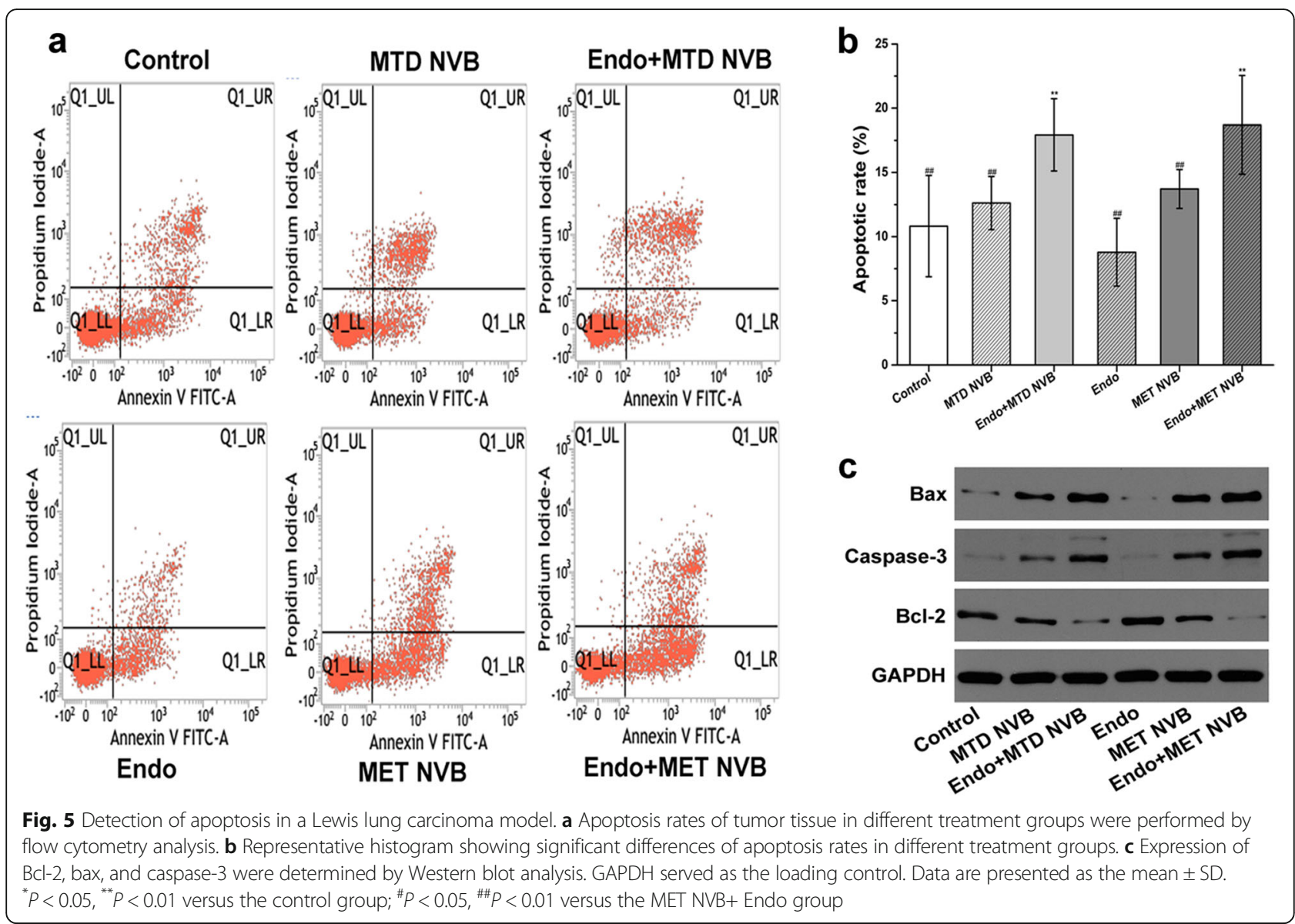

may conceptually produce a synergistic antitumor and anti-angiogenesis effect without overt toxicity, and serve as a promising treatment strategy.

Hypoxia inducible factor 1 (HIF-1) is a hypoxia-regulated transcription factor that modulates the expression of numerous hypoxia-inducible genes [26, 27]. The regulatory activity of HIF-1 is determined by the stability of the HIF- $1 \alpha$ protein, which is stabilized by hypoxia through an $\mathrm{O}_{2}$-dependent degradation domain that rapidly accumulates following exposure to hypoxic conditions [28, 29]. Moreover, hypoxic conditions upregulate the expression of

Table 2 Apoptosis rates of tumor tissue in different treatment groups

\begin{tabular}{llll}
\hline Groups & Bax/GAPDH & Caspase-3/GAPDH & Bcl-2/GAPDH \\
\hline Control & $0.105 \pm 0.001$ & $0.055 \pm 0.002$ & $0.529 \pm 0.002$ \\
MTD NVB & $0.443 \pm 0.002$ & $0.168 \pm 0.001$ & $0.380 \pm 0.001$ \\
Endo+MTD NVB & $0.698 \pm 0.003^{* *}$ & $0.514 \pm 0.002^{* *}$ & $0.148 \pm 0.001^{* *}$ \\
Endo & $0.068 \pm 0.001$ & $0.036 \pm 0.001$ & $0.694 \pm 0.003$ \\
MET NVB & $0.571 \pm 0.002$ & $0.359 \pm 0.001$ & $0.375 \pm 0.001$ \\
Endo+MET NVB & $0.748 \pm 0.003^{* *}$ & $0.602 \pm 0.003^{* *}$ & $0.061 \pm 0.001^{* *}$ \\
\hline
\end{tabular}

Data are presented as the mean \pm SD. ${ }^{*} P<0.05,{ }^{* *} P<0.01$ versus the control group; ${ }^{\#} P<0.05,{ }^{\# \#} P<0.01$ versus the MET NVB+ Endo group
VEGF, thereby promoting tissue permeability and inducing angiogenesis [30, 31]. In tumors, VEGF is the most important angiogenic factor, and inhibits tumor cell apoptosis by inducing the anti-apoptotic protein Bcl-2 [32]. Bcl-2 is an anti-apoptotic protein, and an important modulator of drug-induced apoptosis and chemoresistance [33]. Bax, a pro-apoptotic protein, is found in the cytosol in an inactive form. Caspase- 3 and caspase-7 activation are downstream of many proapoptotic signaling pathways [34]. Under anaerobic conditions, CEPs can rapidly be mobilized and participate in the regeneration of tumor blood vessels. CEPs impact intratumoral blood flow and suppress tumor growth by downregulating $\mathrm{Bcl}-2$ and upregulating expression of bax and caspase $3 / 7$.

Vinorelbine is as an oral formulation and that is convenient to be taken. Previous studies have shown that metronomic oral vinorelbine alone can be safely used in elderly patients with advanced NSCLC $[4,5]$. But in the present study, we investigated the impact of MET NVB and/or Endostar on the frequency of CEPs, expression of CD31, VEGF, and HIF-1 $\alpha$ in tumor-bearing mice. We found that in ctrl-treated mice, peripheral blood CEPs constituted $\sim 0.05 \%$ of circulating blood cells, which resulted in a higher MVD in xenograft tumors. In contrast, 

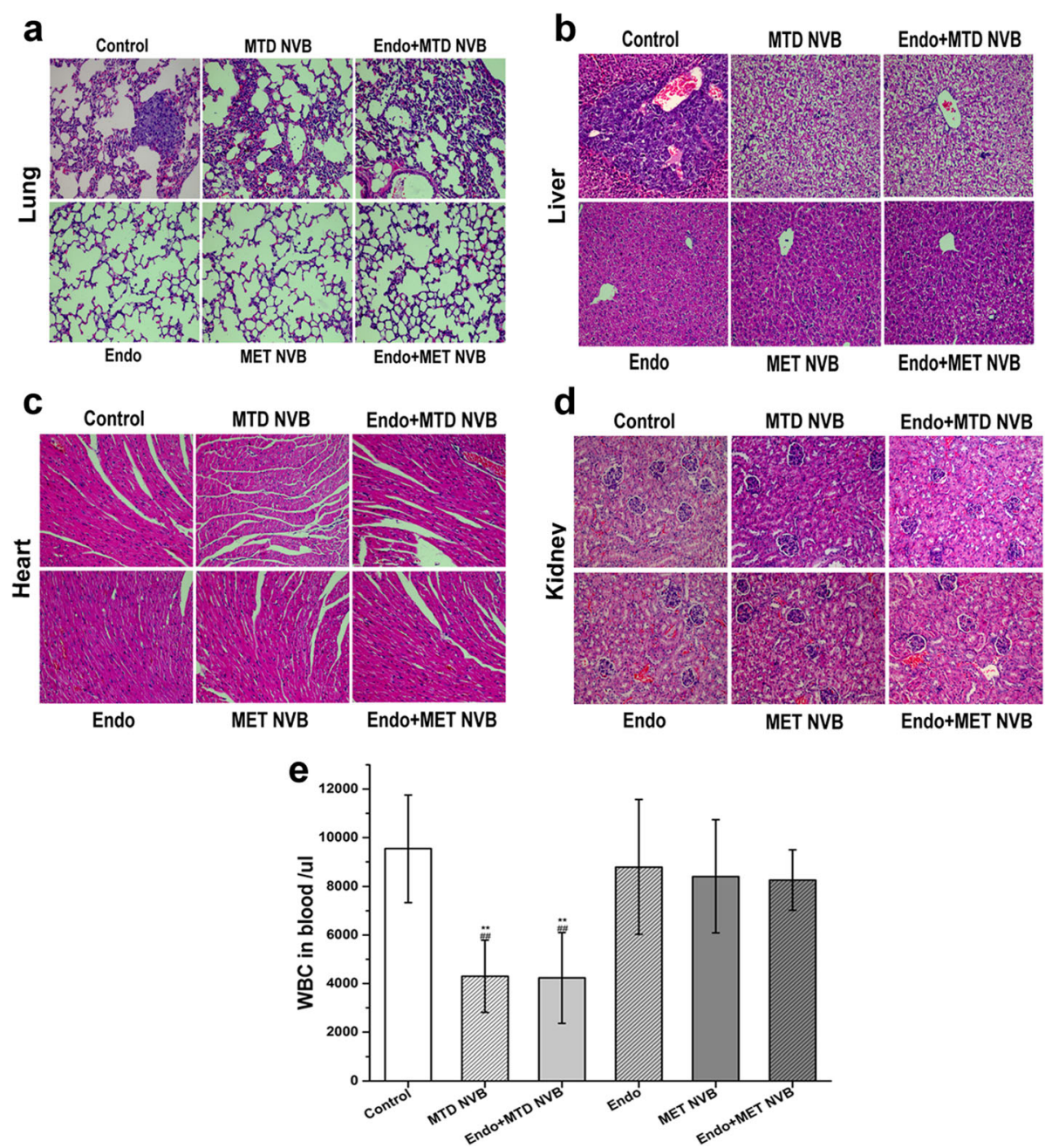

Fig. 6 Evaluation of side effects. a H\&E-stained lung sections of each treatment group (Original magnification, 200). b H\&E-stained liver sections of each treatment group (Original magnification, 200). c H\&E-stained heart sections of each treatment group (Original magnification, 200). d H\&E-stained kidney sections of each treatment group (Original magnification, 200). e Treatment effects on white blood cell (WBC) counts after one full day of treatment. Data are presented as the mean \pm SD. ${ }^{*} P<0.05,{ }^{* *} P<0.01$ versus the control group; ${ }^{\#} P<0.05,{ }^{\# \#} P<0.01$ versus the MET NVB+ Endo group

treatment of MET NVB + Endo markedly reduced the frequency of CEPs, MVD, and the expression of VEGF and HIF- $1 \alpha$. Combining drugs also induced better anti-angiogenic responses compared to monotherapy treatment with either drug. Although we found an increase in the frequency of CEPs, expression of CD31, VEGF and HIF- $1 \alpha$ in both the MTD NVB and MTD $\mathrm{NVB}+$ Endo groups, the differences were not significant. A possible explanation for these findings may be that in cases of high-dose chemotherapy gauge, tumor tissue metabolism and tumor cells are in conditions of hypoxia or insufficient nutrition, and tumor cells promote tumor growth by autophagy degradation and recycling nutrients inside the cell. Anti-angiogenesis therapy may increase tumor hypoxia by promoting tumor cell autophagy and enhancing tumor cell survival ability to cause anti-angiogenesis therapy drug resistance, and vascular formation is promoted by negative feedback regulation. As expected, these findings provide experimental evidence for a synergistic anti-angiogenesis effect of MET and ES, as has recently been shown in a primary tumor model [35].

We also demonstrated that treatment with MTD NVB or/with Endostar effectively inhibited tumor growth and induced apoptosis, but increased tumor vascularity, which is consistent with a role of MTD cytotoxic chemotherapy in inducing endothelial damage [36]. The combination of MET NVB and Endostar significantly enhanced antitumor activity compared to the drugs administered alone, and induced apoptosis by downregulating Bcl-2 expression and upregulating expression of bax and caspase 3/7. In tumor tissue in the control group, a higher apoptosis rate was observed. The reason for this may be that exponential 
cellular proliferation and an inefficient vascular supply leads to increased formation of necrosis in the tumor tissue. Another phenomenon that is often found in solid tumors is that an inadequate oxygen supply results in hypoxic conditions within the tumor. Recent studies have shown that a combination drug administration approach produces no significant synergistic antitumor effects, and that its mechanism of action involves ischemic conditions in solid tumors that can lead to genetic instability and subsequent tumor progression [37, 38]. In contrast, several studies have demonstrated that the administration of combination drugs had improved efficacy compared with drugs that were administered alone. In this regard, several other studies have recently reported circumstances where MET, when using drugs such as cyclophosphamide, can induce vessel normalization, increase perfusion, and transiently decrease the level of tumor hypoxia [39-41]. Moreover, several cytokines, adhesion molecules, and the internal environment caused a corresponding change to achieve the desired antitumor effect by inhibiting the formation of new blood vessels. The results presented in our study were consistent with these findings [35].

Previous studies have suggested that tumor growth and metastasis are inhibited though drugs administered 'metronomically' [3, 21]. However, we investigated whether treatment with Endostar combined with MET NVB or administration of Endostar combined with MTD NVB is superior in regarding antitumor effect. An interesting and unexpected finding was the similar antitumor effect in both treatments. This may be a promising and better approach for the treatment of human cancers. The increased level of CEPs may contribute to the repair of the damaged vasculature after MTD chemotherapy (or plus Endostar) and the decreased level of CEPs suppress the repair and recovery of the tumor vasculature, which is indispensable to tumor growth and metastasis. Regarding the underlying mechanism of action for the opposite effects of MTD NVB or MTD NVB + Endo and MET $\mathrm{NVB}$ on the level of CEPs, a possible explanation may be their opposite effects on the mobilization of CEPs. Mice treated with MTD NVB experienced a robust CEPs mobilization a few days after the end of drug administration, whereas the numbers of CEPs in mice treated with MET NVB were sustained at a very low level for a prolonged period [42, 43].

Previous studies have shown that metronomic topotecan administrated for two weeks compared to the maximum tolerated dose of topotecan enhanced anti-angiogenic responses and had low toxicity used in a xenograft model of retinoblastoma treatment [44]. In our study, these results also indicated that metronomic Vinorelbine combined with Endo administrated for 14 consecutive days had similar results. Cumulative toxicity over a longer period of time may be supplemented in subsequent experiments, but recent toxicity has been observed in this experiment. WBC counts were reduced in the groups administrated MTD $\mathrm{NVB}$, whereas WBC counts were in the normal range in mice administrated MET NVB. Moreover, H\&E-stained sections of the MTD NVB group showed chronic inflammation and interstitial thickening in lung tissue, and hepatic cell edema, degeneration, necrosis, and hepatic structural disorders in liver tissue. These results suggested that Vinorelbine could be an example, which, when given metronomically was not only minimally or non-toxic, but also had little effect when combined with anti-angiogenic agents, and were well compatible with these findings [35].

In the present study, we demonstrated that treatment with MET combined with anti-angiogenesis drugs resulted in robust antitumor effects through enhanced inhibition of tumor-associated angiogenesis, which was consistent with previous findings [45]. It is conceivable that this therapeutic approach can be moved from bench to bedside, particularly for a maintenance therapy in elderly patients with advanced NSCLC to achieve a sustainable tumor control. These patients do not well tolerate side effects, and may benefit by this treatment from a higher quality of life and a longer progression-free survival [46]. However, several studies have reported that anti-angiogenic drugs combined with chemotherapy may exhibit optimal efficacy when administered successively, and that only a short 'time window' for optimal results may exist $[47,48]$. Therefore, further research to address the optimal combination and administration regime of anti-angiogenic and antitumor drugs, whether it may be simultaneous or sequential, is warranted. In addition, the optimal administration plan and suitable treatment doses and frequency of NVB must be determined in further studies and clinical trials.

\section{Conclusions}

In conclusion, this study reports preclinical proof-ofprinciple experiments establishing a rationale for the combination of Endo therapy with MET MVB. In our study, decreasing the expression of CD31, VEGF, and HIF- $1 \alpha$, and peripheral blood CEPs, together with inducing apoptosis and reducing side effects, correlated with the tumor microenvironment and the therapeutic responses to angiogenesis inhibitors, which are promising for uncovering the mechanism of action of anti-angiogenic drugs. Our results emphasized the fact that NVB drugs administered 'metronomically' combined with Endostar resulted in enhanced antitumor and anti-angiogenic effects without overt toxicity in a xenograft model of human lung cancer, and that effects were similar as NVB drugs administered 'standardly' combined with Endostar. These findings may aid in the design of clinical studies to investigate the efficacy and reduced adverse effects of MET combined with an angiogenesis inhibitor for patients with advanced NSCLC, and may serve as an attractive therapeutic modality. 


\section{Abbreviations}

${ }^{18}$ F-FDG PET/CT: fluorine-18-deoxyglucose PET/computed tomography; CEPs: circulating endothelial progenitor cells; HIF-1: Hypoxia inducible factor1; HPF: high power field; MET: Metronomic chemotherapy; MTD: Maximum tolerated dose; MVD: Microvascular density; NSCLC: non-small-cell lung cancer; SUVmax: the maximum of standardized uptake value; VEGF: Vascular epidermal growth factor

\section{Acknowledgements}

The authors thank the members of the Departments of Pathology, Clinical laboratory, and Nuclear Medicine (The Center Laboratory of Southwest Medical University, Luzhou, China), for providing assistance throughout the duration of the study.

\section{Funding}

Research reported in this publication was primarily supported by the China International Medical Foundation (CIMF; No. Z-2014-06-16346) for collection, analysis and interpretation of data. This research was also partially supported during initial stage of the project by the National Natural Science Foundation of China (NSFC; No.81472812) for design of study and initial experiments.

\section{Availability of data and materials}

All data generated or analyzed during this study are included in this published article and its additional files.

\section{Authors' contributions}

RSQ, ZHZ and NPZ performed the study design, animal studies, statistical analysis and drafted the manuscript. HWH and SZF performed the statistical analysis and the animal studies. SSL performed the cell culture. YWH and JF conceived the study, participated in the study design and contributed to draft the manuscript. YC performed micro PET-CT. All authors read and approved the final manuscript.

\section{Ethics approval and consent to participate}

The Committee for Research Ethics and for Animal Care and Use in Research, Southwest Medical University, Luzhou (China) approved the present study. We handled animals in compliance with the revised Animals (Scientific Procedures) Act 1986.

\section{Consent for publication}

Not applicable.

\section{Competing interests}

The authors declare that they have no competing interests.

\section{Publisher's Note}

Springer Nature remains neutral with regard to jurisdictional claims in published maps and institutional affiliations.

\section{Author details}

${ }^{1}$ Suining first people's hospital, Sichuan Province, Suining 629000, China 2 Department of Oncology, the Affiliated Hospital of Southwest Medical University, Sichuan Province, Luzhou 646000, China. ${ }^{3}$ Department of Nuclear Medicine, the Affiliated Hospital of Southwest Medical University, Sichuan Province, Luzhou 646000, China.

Received: 3 April 2018 Accepted: 9 August 2018

Published online: 11 October 2018

\section{References}

1. Kerbel RS, Kamen BA. The anti-angiogenic basis of metronomic chemotherapy. Nat Rev Cancer. 2004:4:423-36.

2. Browder T, Butterfield CE, Kräling BM, et al. Antiangiogenic scheduling of chemotherapy improves efficacy against experimental drug-resistant cancer. Cancer Res. 2000;60(7):1878-86.

3. Pasquier E, Kavallaris M, Andre N. Metronomic chemotherapy: new rationale for new directions. Nat Rev Clin Oncol. 2010;7:455-65.

4. Camerini A, Puccetti C, Amoroso D, et al. Metronomic oral vinorelbine as first-line treatment in elderly patients with advanced non-small cell lung cancer: results of a phase II trial (MOVE trial). BMC Cancer. 2015;15:359.
5. Mencoboni M, Filiberti RA, Taveggia P, et al. Safety of first-line chemotherapy with metronomic single-agent oral Vinorelbine in elderly patients with NSCLC. Anticancer Res. 2017;37(6):3189-94.

6. O'reilly MS, Boehm T, Shing Y, et al. Endostatin: an endogenous inhibitor of angiogenesis and tumor growth. Cell. 1997;88:277-85.

7. Pasquier $\mathrm{E}$, Honore $\mathrm{S}$, Braguer D. Microtubule-targeting agents in angiogenesis: where do we stand? Drug Resist Update. 2006;9:74-86.

8. Soultati A, Mountzios G, Avgerinou C, Papaxoinis G, Pectasides D, et al. Endothelial vascular toxicity from chemotherapeutic agents: Preclinical evidence and clinical implications. Cancer Treat Rev. 2012;38(5):473-83.

9. Briasoulis E, Pappas P, Puozzo C, Tolis C, Fountzilas G, Dafni U, Marselos M Pavlidis N. Dose-ranging study of metronomic oral vinorelbine in patients with advanced refractory cancer. Clin Cancer Res. 2009;15:6454-61.

10. Pastina $P$, Nardone $V$, Croci $S$, et al. Anti-cancer activity of dose-fractioned $\mathrm{mPE}+/$ - bevacizumab regimen is paralleled by immune-modulation in advanced squamous NSLC patients. J Thorac Dis. 2017;9(9):3123-31.

11. Kwak G, Jo SD, Kim D, et al. Synergistic antitumor effects of combination treatment with metronomic doxorubicin and VEGF-targeting RNAi nanoparticles. J Control Release. 2017;267:203-13.

12. Kerbel RS, Shaked Y. The potential clinical promise of 'multimodality' metronomic chemotherapy revealed by preclinical studies of metastatic disease. Cancer Lett. 2017:400:293-304.

13. Ding RL, Xie F, Fu SZ, et al. Preparation of endostatin-loaded chitosan nanoparticles and evaluation of the antitumor effect of such nanoparticles on the Lewis lung cancer model. Drug Deliv. 2017;24(1):300-8.

14. Fan J, Du J, Wu J, et al. Antitumor effects of different administration sequences of cisplatin and Endostar on Lewis lung carcinoma. Oncol Lett. 2015;9(2):822-8.

15. Webb MS, Johnstone S, Morris TJ, Kennedy A, Mayer LD, Bally MB, et al. In vitro and in vivo characterization of a combination chemotherapy formulation consisting of vinorelbine and phosphatidylserine. Eur J Pharm Biopharm. 2007:65(3):289-99.

16. Li C, Cui J, Wang C, et al. Sulfosalicylate mediates improved vinorelbine loading into LUVs and antineoplastic effects. J Liposome Res. 2012;22(1):42-54.

17. Rodríguez-Carrio J, Alperi-López M, López P, Suárez A, et al. Angiogenic T cells are decreased in rheumatoid arthritis patients. Ann Rheum Dis. 2015;74(5):921-7.

18. Rodríguez-Carrio J, Prado C, de Paz B, et al. Circulating endothelial cells and their progenitors in systemic lupus erythematosus and early rheumatoid arthritis patients. Rheumatology. 2012;51(10):1775-84.

19. Otto $S$, Nitsche $K$, Jung $C$, Poerner $T C$, et al. Endothelial progenitor cells and plaque burden in stented coronary artery segments: an optical coherence tomography study six months after elective $\mathrm{PCl}$. BMC Cardiovasc Disord. 2017;17(1):103.

20. Yao W, Firth AL, Sacks RS, Ogawa A, Yuan JX, et al. Identification of putative endothelial progenitor cells (CD34+CD133+Flk-1+) in endarterectomized tissue of patients with chronic thromboembolic pulmonary hypertension. Am J Physiol Lung Cell Mol Physiol. 2009;296(6):L870-8.

21. Francia G, Kerbel RS, Shaked Y, Hashimoto K, Sun J, Yin M, et al. Low-dose metronomic oral dosing of a prodrug of gemcitabine (LY2334737) causes anti-tumor effects in the absence of inhibition of systemic vasculogenesis. Mol Cancer Ther. 2012;11(3):680-9.

22. Yi AH, Miraglia S, Zanjani ED, et al. AC133, a novel marker for human hematopoietic stem and progenitor cells [J]. Blood. 1997;90(12):5002-12.

23. Goon PK, Lip GY, Boos CJ, Stonelake PS, Blann AD. Circulating endothelial cells, endothelial progenitor cells, and endothelial microparticles in cancer. Neoplasia. 2006:8:79-88.

24. Mancuso P, Calleri A, Cassi C, Gobbi A, Capillo M, Pruneri G, Martinelli G, Bertolini F. Circulating endothelial cells as a novel marker of angiogenesis. Adv Exp Med Biol. 2003;522:83-97.

25. Wang J, Sun Y, Liu Y, et al. Results of randomized, multicenter, double-blind phase III trial of rh-endostatin (YH-16) in treatment of advanced non-small cell lung cancer patients. Zhongguo Fei Ai Za Zhi. 2005;8(4):283-90

26. Shannon AM, Bouchier-Hayes DJ, Condron CM, et al. Tumor hypoxia,chemotherapeutic resistance and hypoxia-related therapies. Cancer Treat Rev. 2003:29(4):297-307.

27. Wang GL, Semenza GL. Purification and characterisation of hypoxiainducible factor-1. J Biol Chem. 1995:1:1230-7.

28. Huang LE, Arany Z, Livingston DM, Bunn HF. Activation of hypoxia-inducible transcription factor depends primarily upon redox-sensitive stabilization of its a subunit. J Biol Chem. 1996;271:32252-9. 
29. Huang LE, Jie GU, Schau M, Bunn HF. Regulation of hypoxia-inducible factor-1a is mediated by an O2-dependent degradation domain via the ubiquitinproteasome pathway. Proc Natl Acad Sci U S A. 1998;95:7989-92.

30. Shweiki D, Neeman M, Itin A, Keshet E. Induction of vascular endothelial growth factor expression by hypoxia and by glucose deficiency in multicell spheroids: implications for tumour angiogenesis. Proc Natl Acad Sci U S A. 1995;92:768-72

31. Huang LE, Arany Z, Livingston DM, et al. Activation of hypoxia-inducible transcription factor depends primarily upon redox-sensitive stabilization of its alpha subunit. J Biol Chem. 1996;271(50):32253-9.

32. Pidgeon GP, Barr MP, Harmey JH, Foley DA, Bouchier-Hayes DJ. Vascular endothelial growth factor (VEGF) up regulates $\mathrm{BCl}-2$ and inhibits apoptosis in human and murine mammary adenocarcinoma cells. Br J Cancer. 2001;85:273-8.

33. Fei ZW, Qiu MK, Qi XQ, Ou JM, et al. Oxymatrine suppresses proliferation and induces apoptosis of hemangioma cells through inhibition of HIF-1a signaling. Int J Immunopathol Pharmacol. 2015;28(2):201-8.

34. Karaca B, Atmaca H, Bozkurt E, Uslu R, et al. Combination of AT-101/cisplatin overcomes chemoresistance by inducing apoptosis and modulating epigenetics in human ovarian cancer cells. Mol Biol Rep. 2013;40(6):3925-33.

35. Srivastava K, Hu J, Korn C, et al. Postsurgical adjuvant tumor therapy by combining anti-angiopoietin-2 and metronomic chemotherapy limits metastatic growth. Cancer Cell. 2014;26(6):880-95.

36. Laquente B, Vinals F, Germa JR. Metronomic chemotherapy: an antiangiogenic scheduling. Clin Transl Oncol. 2007;9:93-8.

37. Bindra RS, Glazer PM. Genetic instability and the tumor microenvironment: towards the concept of microenvironment-induced mutagenesis. Mutat Res. 2005;569(1-2):75-85.

38. Huang LE, Bindra RS, Glazer PM, Harris AL. Hypoxia-induced genetic instability-a calculated mechanism underlying tumor progression. J Mol Med. 2007;85(2):139-48.

39. Mupparaju S, Hou H, Lariviere JP, Swartz HM, Khan N. Tumor pO as a surrogate marker to identify therapeutic window during metronomic chemotherapy of 9L gliomas. Adv Exp Med Biol. 2011;701:107-13.

40. Doloff JC, Khan N, Ma J, et al. Increased tumor oxygenation and drug uptake during anti-angiogenic weekly low dose cyclophosphamide enhances the antitumor effect of weekly tirapazamine. Curr Cancer Drug Targets. 2009;9:777-88.

41. Cham KK, Baker JH, Takhar KS, et al. Metronomic gemcitabine suppresses tumour growth, improves perfusion, and reduces hypoxia in human pancreatic ductal adenocarcinoma. Br J Cancer. 2010;103:52-60.

42. Bertolini F, Paul S, Mancuso P, et al. Maximum tolerable dose and lowdose metronomic chemotherapy have opposite effects on the mobilization and viability of circulating endothelial progenitor cells. Cancer Res. 2003;63(15):4342-6.

43. Goon PK, Lip GY, Stonelake PS, Blann AD. Circulating endothelial cells and circulating progenitor cells in breast cancer: relationship to endothelial damage/dysfunction/apoptosis, clinicopathologic factors, and the Nottingham prognostic index. Neoplasia. 2009;11:771-9.

44. Winter U, Mena HA, Negrotto S, et al. Schedule-dependent antiangiogenic and cytotoxic effects of chemotherapy on vascular endothelial and retinoblastoma cells. PLoS One. 2016;11:7.

45. Pietras K, Hanahan D. A multitargeted, metronomic, and maximumtolerated dose 'chemo-switch' regimen is antian-giogenic, producing objective responses and survival benefit in a mouse model of cancer. J Clin Oncol. 2005;23:939-52.

46. Fidias P And Novello S. Strategies for prolonged therapy in patients.with advanced non-small-cell lung cancer. J Clin Oncol.2010; 28: 5116-5123.

47. Zhou ZT, Zhou FX, Wei Q, Zou LY, Qin BF, Peng XS. Phase II study of cisplatin/etoposide and endostar for extensive-stage small-cell lung cancer. Cancer Chemother Pharmacol. 2011;68:1027-32

48. Ma X, Yao Y, Yuan D, Liu H, Wang S, Zhou C, Song Y. Recombinant human endostatin endostar suppresses angio-genesis and lymphangiogenesis of malignant pleural effusion in mice. PLoS One. 2012;7:e53449.

\section{Ready to submit your research? Choose BMC and benefit from:}

- fast, convenient online submission

- thorough peer review by experienced researchers in your field

- rapid publication on acceptance

- support for research data, including large and complex data types

- gold Open Access which fosters wider collaboration and increased citations

- maximum visibility for your research: over $100 \mathrm{M}$ website views per year

At BMC, research is always in progress.

Learn more biomedcentral.com/submissions 